\title{
Evaluations of Different Montage Types and Sizes on Eri and Mulberry Feeding Silkworms Cocoon Yield and Quality of Silk, at Melkassa Agricultural Research Center, East Shoa, Ethiopia
}

\author{
Ahmed Ibrahim*, Kedir Shifa, Abiy Tilahun and Metasebia Terefe \\ Ethiopian Institute of Agricultural Research, Melkassa Agricultural Research Center, P.O. Box: 436, \\ Melkassa, Ethiopia
}

\begin{tabular}{|c|c|}
\hline Abstract & Article Inforn \\
\hline 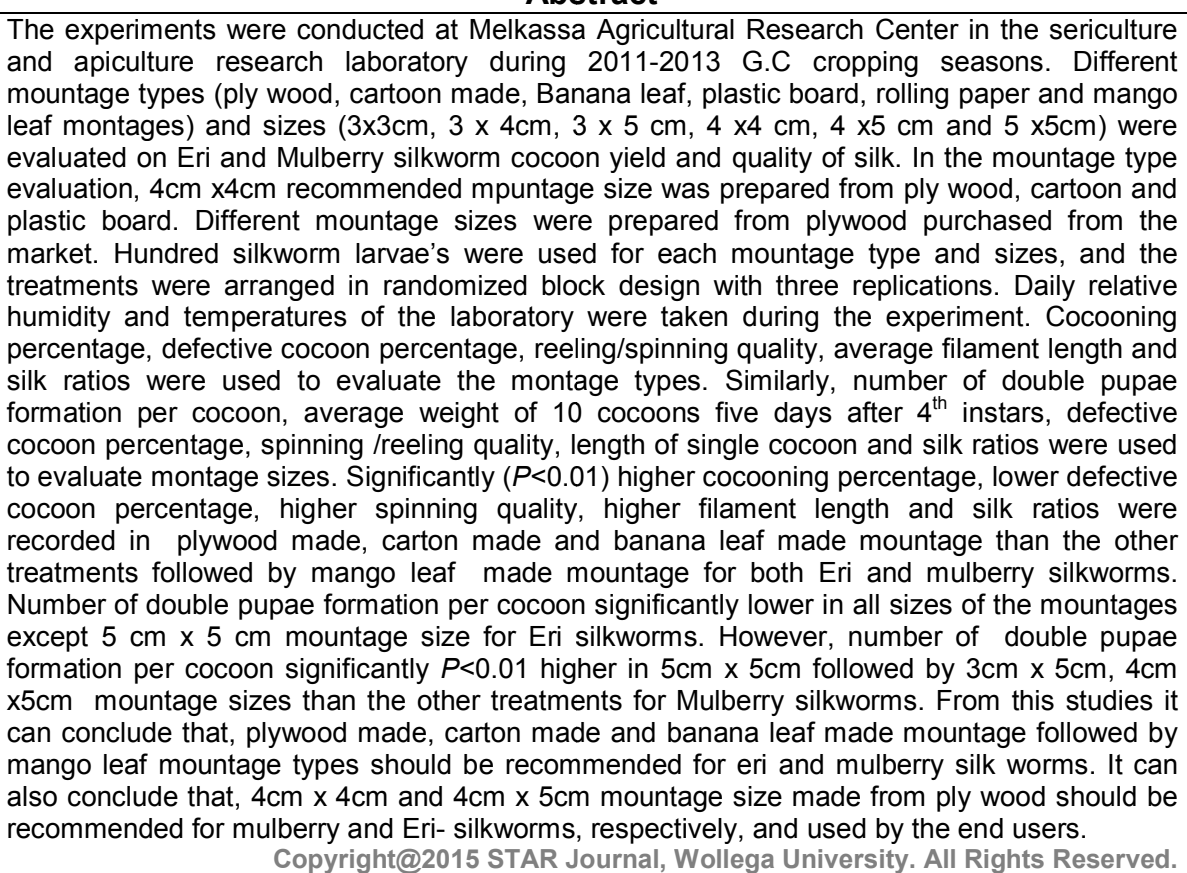 & Received : 01-03-2015 \\
\hline
\end{tabular}

\section{INTRODUCTION}

Mounting is the last stage of rearing operation. Transferring mature fifth instar larvae to mountages is called mounting. When larvae are fully mature, they become translucent, their body shrinks, and they stop feeding and start searching for suitable place to attach themselves for cocoon spinning and pupation. These movements clearly indicate to transfer the mature larvae into the mountages.

They are picked up and put on mountages. The worms attach themselves to the spirals of the mountages and start spinning the cocoon. By continuous movement of head, silk fluid is released in minute quantity which hardens to form a long continuous filament. The silkworm at first lays the foundation for the cocoon structure by weaving a preliminary web providing the necessary foot hold for the larva to spin the compact shell of cocoon.
Owing to characteristic movements of the head, the silk filament is deposited in a series of short waves forming the figure of eight. This way layers are built and added to form the compact cocoon shell. After the compact shell of the cocoon is formed, the shrinking larva wraps itself and detaches from the shell and becomes pupa or chrysalis. The spinning completes within 2-3 days in multi-voltine varieties and 3-4 days in uni- and bivoltine.

Mountages types which are having proper sizes play a vital role in quality cocoon production. Farmers depend on resources and use different types of materials available locally for making Mountages. Types of material used, finishing of Mountages, space available for spinning worms in Mountages etc., will decide the quality of cocoon. Narrow space affects ventilation and results in 
Ahmed lbrahim et al.,

poor reliability of cocoons. Similarly more space results in wastage of silk in the form of floss (Mathur et. al, 2010).

Different types of mountages are used in different parts of silk producing areas of the world. In addition to support the spinning worms, the mountages should satisfy the requirements like, it provide convenient space of suitable dimension for spinning good sized cocoons, should not promote formation of double cocoons, malformed cocoons and flimsy cocoons, should have provisions for drying up of the last excreta of the worm prior to spinning and prevention of its falling on the cocoons of other worms, should be suitable for easy mounting and harvesting.

The material and structure of montages significantly affect the quality of cocoon filament and also the labor required for mounting and harvesting the cocoons. The basic concept of proper montages types and sizes are to provide an angular uniform space for silk worm to facilitate easy cocoon formation. The fabrication and type of montages depends on the availability of chief materials in the respective places. If the material and structure of montages are not proper, it will affect the shape and size of cocoons, besides increasing of double, deformed, soiled cocoons and wastages of silk in the form of floss. The common montages used at present in India and China are made out of bamboo, plastic material, wood etc. However, the works on mountage types and size are scanty in Ethiopia. Therefore, it is very crucial to evaluate different types of montages types and size for better cocoon yield and silk quality for our case.

\section{MATERIALS AND METHODS}

\section{Description of Study Area}

The experiments were conducted at Melkassa Agricultural research center, for two years (2012 and 2013 G.C.). The place lies at $8^{\circ} 24^{\prime} \mathrm{N}$ latitude and $39^{\circ} 21^{\prime} \mathrm{E}$ longitude, $17 \mathrm{~km}$ south of Adama, Nazareth, at an altitude of 1550 m.a.s.l. The area is characterized by warm and semi-humid climate. The annual average rainfall and relative humidity of the laboratory during 2012 and 2013 G.C. cropping seasons are $810.1 \mathrm{~mm}, 924.7 \mathrm{~mm}$ and 50rh, $55 \mathrm{rh}$, respectively. The average minimum and maximum atmospheric temperatures of the laboratory during 2012 and 2013 G.C. cropping seasons are 9.3, 13.2 and 28.9, 29.1 , respectively.

\section{Experimental Design and Methods}

The experiment was conducted for two years, i.e. during the 2012, 2013G.C. cropping reasons. Six different montage types (ply wood, cartoon made, Banana leaf made, plastic, rolling paper and mango leaf montages) and six different montage sizes $(3 \times 3,3 \times 4,3 \times 5,4 \times 4,4 \times 5$ and $5 \times 5 \mathrm{~cm}$ ) were evaluated. The experiment was laid out in complete randomized design with four replications during 2012 and 2013 seasons experiment. For the montage type activity, ply wood, cartoon and plastic made mountage were purchased from the market and montages were made in the center. Banana and mango branch with leaf were collected from Horticultural Research Center of Melkassa Agricultural Research Center and dried under shade and used as montage for the silkworm. Regarding the montage size experiment, ply wood was purchased from the market and montages were prepared which are having different sizes. Cocooning percentage, defective cocoon percentage, reliability, average filament length and silk ratios were used to evaluate the montage types.
Sci. Technol. Arts Res. J., April-June 2015, 4(2): 48-52

Similarly, number of double cocoon formation/plot, average weight of 10 cocoons, defective cocoon percentage, spinning quality, length of single cocoon and silk ratios were used for the evaluation of montage sizes. Data were subjected to SAS 6.12 soft ware.

\section{RESULTS AND DISCUSSION}

The effects of different mountage types on silkworm cocooning percentage, defective coccon percentage, spinning quality, filament length and silk ratios of Eri-3.4 are indicated in Table1and Table2. Significantly $(P<0.01)$ higher cocooning percentage, lower defective cocoon percentage, higher spinning quality and higher filament length were recorded in plywood, carton made and banana leaf made mountage followed by mango leaf made mountage (Table 1 and Table 2). Even though lower cocooning percentage and higher defective cocoon percentage were observed in plastic made mountage, significantly higher spinning quality, higher average filament length and silk ratios were observed (Table 1). On the other hands, silk ratios for all the treatments were in the acceptable ranges for both Eri-India and Eri-3.4 silkworms, except for rolling paper treatment of Eri-3.4 silkworms (Table 1). Significantly higher cocooning percentage, reeling quality and lower defective cocoon percentage were observed in plywood, carton made and banana leaf made mountage followed by the other mountage types for both Korean and Kenyan silkworms and non significant differences were recorded among the treatments with in the column (Table 4). An average filament length and silk ratios were significantly higher in all the mountage types and insignificant differences were observed among them with in the column for both Korean and Kenian bivoltine (Table 3 and Table4). Similarly, cocooning percentage significantly higher in cartoon and banana leaf made mountage followed by ply wood mountage for mulberry multivoltine silkworms (Table 5). Defective cocoon percentages significantly higher in plastic made mountage followed by rolling paper mountage but significantly lower in the other treatments. An average filament length significantly $(P<0.01)$ higher in the plywood, cartoon, banana leaf and plastic made mountages than the other treatments and non significant differences were observed among them. But, significant differences were recorded among the treatments with respect to the silk ratios; however, all the values recorded were in the acceptable ranges for mulberry multivoltine silkworms (Table 5).

The effects of different mountage sizes of plywood on the number of pupae /cocoon/plot, weight of cocoon, defective cocoon percentage, spinning quality, length of single cocoon and silk ratios of Eri- 3.4 and Eri India silkworm are indicated in Table 6 and Table 7. Significant differences were observed among the treatments within the column for all parameters. Number of double pupae/cocoon/ plot significantly lower in all sizes of the mountages, except for $5 \mathrm{~cm} \times 5 \mathrm{~cm}$ mountage size. An average weight of 10 cocoons and length of single cocoon significantly lower in the $3 \mathrm{~cm} \times 3 \mathrm{~cm}$ and $4 \mathrm{~cm} \times 3 \mathrm{~cm}$ but significantly higher in the other treatments and non significant differences were observed among them within the column. On the other hands, insignificant and similar results were observed among the treatments within the column for defective cocoon percentages, spinning quality and silk ratios of different mountage sizes (Table 6). On the other hands, number of double pupae/cocoon/plot significantly $(P<0.01)$ higher in $5 \mathrm{~cm} \times 5 \mathrm{~cm}$ followed by 
Ahmed lbrahim et al.,

$4 \mathrm{~cm} \times 5 \mathrm{~cm}$ and $3 \mathrm{~cm} \times 5 \mathrm{~cm}$ mountage sizes (Table 7 ). However, significantly lower numbers of double pupae/cocoons/plot formation were appeared in the other mountage sizes. Similarly, insignificant and higher average weight of 10 cocoons were observed among the mountgae sizes. Defective cocoon percentage and length of spinning thread significantly higher in $3 \mathrm{~cm} 5 \mathrm{~cm}, 4 \mathrm{~cm}$
Sci. Technol. Arts Res. J., April-June 2015, 4(2): 48-52

$x 5 \mathrm{~cm}$ and $5 \mathrm{~cm} \times 5 \mathrm{~cm}$ mountage sizes than the other treatments. Nevertheless, the reeling quality and the silk ratios were significantly higher in $3 \mathrm{~cm} \times 3 \mathrm{~cm}, 3 \mathrm{~cm} \times 4 \mathrm{~cm}$ and $4 \mathrm{~cm} \times 4 \mathrm{~cm}$ mountgae sizes and non significant differences were recorded among them within their columns (Table 7).

Tabele 1: The effects of different mountage types on silkworm cocooning percentage, defective coccon percentage, spinning quality filament length and silk ratios of Eri-3.4 silkworms

\begin{tabular}{clccccc}
\hline No & Treatments & $\begin{array}{c}\text { Cocooning } \\
\text { percentage } \\
(\%)\end{array}$ & $\begin{array}{c}\text { Defective } \\
\text { cocoon } \\
\text { percentage (\%) }\end{array}$ & $\begin{array}{c}\text { Spinning } \\
\text { quality (\%) }\end{array}$ & $\begin{array}{c}\text { Average filament } \\
\text { length of 10 } \\
\text { cocoons (M) }\end{array}$ & $\begin{array}{c}\text { Silk } \\
\text { ratio (\%) }\end{array}$ \\
\hline 1 & $\begin{array}{l}\text { Ply wood made } \\
\text { mountage }\end{array}$ & $97.20 \pm 0.44 \mathrm{a}$ & $0.012 \pm 0.01 \mathrm{c}$ & $96.87 \pm 0.62 \mathrm{a}$ & $8.88 \pm 0.32 \mathrm{a}$ & $11.53 \pm 0.22 \mathrm{ab}$ \\
2 & $\begin{array}{l}\text { carton made } \\
\text { maountage }\end{array}$ & $95.69 \pm 0.56 \mathrm{a}$ & $0.014 \pm 0.01 \mathrm{c}$ & $96.65 \pm 1.01 \mathrm{a}$ & $8.36 \pm 0.64 \mathrm{ab}$ & $11.44 \pm 0.04 \mathrm{~b}$ \\
3 & $\begin{array}{l}\text { Banana leaf made } \\
\text { mountage }\end{array}$ & $96.69 \pm 0.22 \mathrm{a}$ & $0.015 \pm 0.01 \mathrm{c}$ & $95.63 \pm 0.68 \mathrm{a}$ & $7.86 \pm 0.13 \mathrm{cb}$ & $11.77 \pm 0.18 \mathrm{a}$ \\
4 & $\begin{array}{l}\text { Plastic made } \\
\text { mountage }\end{array}$ & $69.01 \pm 0.50 \mathrm{c}$ & $0.459 \pm 0.02 \mathrm{a}$ & $91.74 \pm 5.75 \mathrm{a}$ & $8.34 \pm 0.16 \mathrm{ab}$ & $11.42 \pm 0.20 \mathrm{ab}$ \\
5 & $\begin{array}{l}\text { Rolling paper } \\
\text { mountage }\end{array}$ & $70.84 \pm 3.76 \mathrm{c}$ & $0.473 \pm 0.01 \mathrm{a}$ & $65.01 \pm 4.40 \mathrm{~b}$ & $6.63 \pm 0.11 \mathrm{~d}$ & $9.74 \pm 0.22 \mathrm{c}$ \\
6 & $\begin{array}{l}\text { Mango leaf made } \\
\text { mountage }\end{array}$ & $80.50 \pm 5.10 \mathrm{~b}$ & $0.050 \pm 0.01 \mathrm{~b}$ & $97.46 \pm 0.99 \mathrm{a}$ & $6.93 \pm 0.09 \mathrm{~cd}$ & $11.41 \pm 0.17 \mathrm{ab}$ \\
\hline \multicolumn{2}{c}{$\mathbf{C V} \%$} & $\mathbf{5 . 3 3}$ & $\mathbf{9 . 3 5}$ & $\mathbf{6 . 2 6}$ & $\mathbf{6 . 5 8}$ & $\mathbf{3 . 0 8}$ \\
\hline
\end{tabular}

(Student-Newman-Keul`s Range Test).

Table 2: The effects of different mountage types on silk worm cocooning percentage, defective cocooning percentage, spinning quality, filament length and silk ratios of Eri - India mixed silkworms

\begin{tabular}{|c|c|c|c|c|c|c|}
\hline No. & Treatments & $\begin{array}{c}\text { Cocooning } \\
\text { percentage } \\
(\%) \\
\end{array}$ & $\begin{array}{c}\text { Defective } \\
\text { cocoon } \\
\text { percentage (\%) } \\
\end{array}$ & $\begin{array}{l}\text { Spinning } \\
\text { quality }(\%)\end{array}$ & $\begin{array}{c}\text { Average filament } \\
\text { length of } \\
10 \text { cocoons (M) }\end{array}$ & $\begin{array}{l}\text { Silk } \\
\text { ratio (\%) }\end{array}$ \\
\hline 1 & $\begin{array}{l}\text { Ply wood made } \\
\text { mountage }\end{array}$ & $93.26 \pm 0.75 a$ & $0.088 \pm 0.01 \mathrm{c}$ & $85.78 \pm 0.78 a$ & $8.09 \pm 0.13 a$ & $12.38 \pm 0.08 a$ \\
\hline 2 & $\begin{array}{l}\text { carton made } \\
\text { maountage }\end{array}$ & $93.70 \pm 0.42 a$ & $0.105 \pm 0.01 \mathrm{c}$ & $86.83 \pm 0.44 a$ & $8.24 \pm 0.22 a$ & $12.05 \pm 0.18 a$ \\
\hline 3 & $\begin{array}{l}\text { Banana leaf made } \\
\text { mountage }\end{array}$ & $92.11 \pm 0.63 a$ & $0.094 \pm 0.01 \mathrm{c}$ & $86.15 \pm 0.11 a$ & $7.92 \pm 0.12 \mathrm{ab}$ & $11.87 \pm 0.39 a$ \\
\hline 4 & $\begin{array}{l}\text { Plastic made } \\
\text { mountage }\end{array}$ & $67.09 \pm 1.30 c$ & $0.332 \pm 0.02 b$ & $76.67 \pm 2.31 b$ & $8.19 \pm 0.18 a$ & $11.83 \pm 0.13 a$ \\
\hline 5 & $\begin{array}{l}\text { Rolling paper } \\
\text { mountage }\end{array}$ & $62.93 \pm 1.73 d$ & $0.447 \pm 0.03 a$ & $62.91 \pm 2.50 c$ & $7.55 \pm 0.29 b$ & $11.12 \pm 0.34 b$ \\
\hline 6 & $\begin{array}{l}\text { Mango leaf made } \\
\text { mountage }\end{array}$ & $82.59 \pm 0.28 b$ & $0.124 \pm 0.02 \mathrm{c}$ & $88.07 \pm 0.13 a$ & $6.76 \pm 0.26 c$ & $10.76 \pm 0.08 b$ \\
\hline & CV\% & 1.76 & 13.05 & 2.69 & 3.97 & 3.28 \\
\hline
\end{tabular}

(Student-Newman-Keul’s Range Test)

Table 3: The effects of different mountage types on silkworm cocooning percentage, defective cocoon percentage, reeling quality, filament length and silk ratios of Kenya bivoltine (K1, K3, K4 and K5) silkworms

\begin{tabular}{|c|c|c|c|c|c|c|}
\hline No & Treatments & $\begin{array}{c}\text { Cocooning } \\
\text { percentage (\%) }\end{array}$ & $\begin{array}{c}\text { Defective } \\
\text { cocoon } \\
\text { percentage (\%) }\end{array}$ & $\begin{array}{c}\text { Reeling } \\
\text { quality (\%) }\end{array}$ & $\begin{array}{l}\text { Average filament } \\
\text { length of } 10 \\
\text { cocoons }(\mathrm{m})\end{array}$ & $\begin{array}{c}\text { Silk } \\
\text { ratio (\%) }\end{array}$ \\
\hline 1 & $\begin{array}{l}\text { Ply wood made } \\
\text { mountage }\end{array}$ & $95.31 \pm 1.21 \mathrm{a}$ & $0.020 \pm 0.001 \mathrm{c}$ & 83.53+1.76ab & $811.90 \pm 12.87 \mathrm{a}$ & $21.87 \pm 0.49 a$ \\
\hline 2 & $\begin{array}{l}\text { carton made } \\
\text { maountage }\end{array}$ & $87.45+5.31 \mathrm{a}$ & $0.025 \pm 0.001 \mathrm{c}$ & $82.31 \pm 2.00 \mathrm{ab}$ & $827.45+20.95 a$ & $23.74 \pm 0.04 a$ \\
\hline 3 & $\begin{array}{l}\text { Banana leaf made } \\
\text { mountage }\end{array}$ & $87.88 \pm 0.92 a$ & $0.032 \pm 0.001 \mathrm{c}$ & $81.20 \pm 1.64 a b$ & $830.85 \pm 45.20 a$ & $22.46 \pm 1.17 a$ \\
\hline 4 & $\begin{array}{l}\text { Plastic made } \\
\text { mountage }\end{array}$ & $62.19 \pm 3.49 b$ & $0.131 \pm 0.001 b$ & $86.76 \pm 0.23 a$ & $797.44 \pm 10.39 a$ & $21.39 \pm 0.41 \mathrm{ab}$ \\
\hline 5 & $\begin{array}{l}\text { Rolling paper } \\
\text { mountage }\end{array}$ & $64.47 \pm 5.09 b$ & $0.351 \pm 0.026 a$ & $64.63 \pm 3.92 \mathrm{c}$ & $851.49 \pm 56.00 a$ & $19.04 \pm 1.14 b$ \\
\hline 6 & $\begin{array}{l}\text { Mango leaf made } \\
\text { mountage }\end{array}$ & $57.83 \pm 2.58 \mathrm{~b}$ & $0.017 \pm 0.001 \mathrm{c}$ & $79.81 \pm 2.45 b$ & $805.73 \pm 15.01 a$ & $22.91+0.17 a$ \\
\hline & CV\% & 8.44 & 19.79 & 4.67 & 7.36 & 6.23 \\
\hline
\end{tabular}

Means followed by the same letter within a column are not significantly different from each other at $1 \%$ level of probability

(Student-Newman-Keul's Range Test). 
Table 4: The effects of different mountage types, on silkworm cocooning percentage, defective cocoon percentage, reeling quality, filament length and silk ratios of Korea- bivoltine silkworms

\begin{tabular}{|c|c|c|c|c|c|c|}
\hline No. & Treatments & $\begin{array}{c}\text { Cocooning } \\
\text { percentage } \\
(\%)\end{array}$ & $\begin{array}{c}\text { Defective } \\
\text { cocoon } \\
\text { percentage (\%) }\end{array}$ & $\begin{array}{c}\text { Reeling } \\
\text { quality (\%) }\end{array}$ & $\begin{array}{l}\text { Avarage filament } \\
\text { length of } 10 \\
\text { cocoons (M) }\end{array}$ & $\begin{array}{c}\text { Silk } \\
\text { ratio (\%) }\end{array}$ \\
\hline 1 & $\begin{array}{l}\text { Ply wood made } \\
\text { mountage }\end{array}$ & $75.71 \pm 1.18 a$ & $1.34 \pm 0.06 b$ & $73.68 \pm 2.07 a b$ & $701.67 \pm 2.33 a$ & $21.43 \pm 0.57 b$ \\
\hline 2 & $\begin{array}{l}\text { carton made } \\
\text { maountage }\end{array}$ & $74.08 \pm 0.57 a$ & $1.23 \pm 0.07 b$ & $74.60 \pm 0.77 a$ & $709.00 \pm 1.00 a$ & $23.15 \pm 0.44 a$ \\
\hline 3 & $\begin{array}{l}\text { Banana leaf made } \\
\text { mountage }\end{array}$ & $71.61 \pm 1.08 a$ & $1.34 \pm 0.04 b$ & $74.15 \pm 0.58 a$ & $707.44 \pm 4.61 a$ & $22.21 \pm 0.48 a b$ \\
\hline 4 & $\begin{array}{l}\text { Plastic made } \\
\text { mountage }\end{array}$ & $57.21+4.02 \mathrm{c}$ & $2.59 \pm 0.25 a$ & $65.28 \pm 5.89 b c$ & $575.81 \pm 17.01 b$ & $22.91 \pm 0.36 a b$ \\
\hline 5 & $\begin{array}{l}\text { Rolling paper } \\
\text { mountage }\end{array}$ & $74.71 \pm 0.35 a$ & $2.58 \pm 0.26 a$ & $59.72 \pm 2.86 c$ & $574.34 \pm 20.57 b$ & $23.80 \pm 0.16 a$ \\
\hline 6 & $\begin{array}{l}\text { Mango leaf made } \\
\text { maountage }\end{array}$ & $65.46 \pm 1.39 b$ & $1.31 \pm 0.10 b$ & $75.05 \pm 1.01 a$ & $707.89 \pm 2.08 a$ & $22.33 \pm 0.16 a b$ \\
\hline & CV\% & 4.74 & 15.02 & 6.68 & 3.14 & 4.00 \\
\hline
\end{tabular}

Table 5: The effects of different mountage types on silkworm cocooning percentage, defective cocoon percentage, reeling quality, filament length and silk ratios of Multivoltine silkworm

\begin{tabular}{|c|c|c|c|c|c|c|}
\hline No & Treatments & $\begin{array}{c}\text { Cocooning } \\
\text { percentage (\%) }\end{array}$ & $\begin{array}{c}\text { Defective } \\
\text { cocoon } \\
\text { percentage (\%) }\end{array}$ & $\begin{array}{c}\text { Reeling } \\
\text { quality (\%) }\end{array}$ & $\begin{array}{c}\text { Average filament } \\
\text { length of } 10 \text { cocoons }(\mathrm{m})\end{array}$ & $\begin{array}{c}\text { Silk } \\
\text { ratio (\%) }\end{array}$ \\
\hline 1 & $\begin{array}{l}\text { Ply wood made } \\
\text { mountage }\end{array}$ & $89.81 \pm 0.75 b$ & $0.128 \pm 0.005 d$ & $83.91 \pm 1.52 a$ & $825.57 \pm 31.35 a b c$ & $21.61 \pm 0.32 c$ \\
\hline 2 & $\begin{array}{l}\text { Carton made } \\
\text { maountage }\end{array}$ & $91.21+0.14 a b$ & $0.190+0.007 \mathrm{c}$ & $84.13 \pm 1.52 a$ & $877.17 \pm 4.34 a$ & $23.28+0.28 a$ \\
\hline 3 & $\begin{array}{l}\text { Banana leaf made } \\
\text { mountage }\end{array}$ & $93.51+1.50 a$ & $0.175+0.006 c$ & $83.10+1.00 a$ & $815.02+15.50 \mathrm{bc}$ & $23.38+0.30 a$ \\
\hline 4 & $\begin{array}{l}\text { Plastic made } \\
\text { mountage }\end{array}$ & $70.13 \pm 0.80 d$ & $0.423 \pm 0.004 a$ & $67.84 \pm 3.93 b$ & $834.33 \pm 18.18 a b$ & $22.46 \pm 0.56 a b$ \\
\hline 5 & $\begin{array}{l}\text { Rolling paper } \\
\text { mountage }\end{array}$ & $62.89 \pm 0.92 \mathrm{e}$ & $0.368+0.013 b$ & $63.13 \pm 2.25 b$ & $638.04 \pm 13.76 \mathrm{~d}$ & $20.62 \pm 0.28 c$ \\
\hline \multirow[t]{2}{*}{6} & $\begin{array}{l}\text { Mango leaf made } \\
\text { mountage }\end{array}$ & $73.94 \pm 0.58 \mathrm{c}$ & $0.138 \pm 0.001 d$ & $81.39 \pm 1.37 a$ & $768.33 \pm 10.80 \mathrm{c}$ & $22.59 \pm 0.38 a b$ \\
\hline & CV\% & 1.84 & 5.84 & 5.24 & 4.14 & 3.01 \\
\hline
\end{tabular}

Table 6: Effects of different mountage sizes of plywood on number of cocoon /single space, weight of cocoon, defective cocoon percentage, spinning quality, length of single cocoon and silk ratios of Eri- 3.4 and India silkworm

\begin{tabular}{|c|c|c|c|c|c|c|c|}
\hline No & Treatments & $\begin{array}{l}\text { Number of } \\
\text { double and } \\
\text { above/plot }\end{array}$ & $\begin{array}{c}\text { Average } \\
\text { weight of } 10 \\
\text { cocoons }\end{array}$ & $\begin{array}{c}\text { Defective } \\
\text { cocoon } \\
\text { percentage (\%) }\end{array}$ & $\begin{array}{l}\text { Spinning } \\
\text { quality (\%) }\end{array}$ & $\begin{array}{l}\text { Length of a } \\
\text { single cocoons }\end{array}$ & $\begin{array}{c}\text { Silk } \\
\text { ratio (\%) }\end{array}$ \\
\hline 1 & $3 \mathrm{~cm} \times 3 \mathrm{~cm}$ & $0.00 \pm 0.00 b$ & $2.46 \pm 0.22 c$ & $0.00 \pm 0.00 a$ & $76.53 \pm 1.94 a$ & $5.803 \pm 0.52 c$ & $11.63 \pm 0.33 a$ \\
\hline 2 & $3 \mathrm{~cm} \times 4 \mathrm{~cm}$ & $0.00 \pm 0.00 b$ & $3.30 \pm 0.02 b$ & $0.00 \pm 0.00 a$ & $73.58 \pm 1.31 a$ & $7.660 \pm 0.11 b$ & $11.95 \pm 0.12 a$ \\
\hline 3 & $3 \mathrm{~cm} \times 5 \mathrm{~cm}$ & $0.00 \pm 0.00 b$ & $3.92 \pm 0.09 a$ & $0.00 \pm 0.00 a$ & $74.01+2.13 a$ & $8.380 \pm 0.14 a$ & $12.20 \pm 0.49 a$ \\
\hline 4 & $4 \mathrm{~cm} \times 4 \mathrm{~cm}$ & $0.00 \pm 0.00 b$ & $3.79 \pm 0.03 a$ & $0.00 \pm 0.00 a$ & $74.74 \pm 0.37 a$ & $7.667 \pm 0.08 b$ & $12.90 \pm 0.95 a$ \\
\hline 5 & $4 \mathrm{~cm} \times 5 \mathrm{~cm}$ & $0.00 \pm 0.00 b$ & $4.07 \pm 0.02 a$ & $0.00 \pm 0.00 a$ & $74.43 \pm 0.85 a$ & $8.086 \pm 0.04 a b$ & $12.23 \pm 0.88 a$ \\
\hline 6 & $5 \mathrm{~cm} \times 5 \mathrm{~cm}$ & $2.05 \pm 0.00 a$ & $4.08 \pm 0.07 a$ & $0.00 \pm 0.00 a$ & $73.81 \pm 1.52 a$ & $8.106 \pm 0.02 a b$ & $11.68 \pm 0.27 a$ \\
\hline & CV\% & 5.34 & 5.34 & -- & 3.74 & 5.01 & 7.77 \\
\hline
\end{tabular}
(Student-Newman-Keul's Range Test).

Table 7: Effects of different mountage sizes of plywood on number of cocoon /single space, weight of cocoon, defective cocoon percentage, reeling quality, length of single cocoon and silk ratios of bivoltine and multivoltine silkworms (K1, K3, K4, K5, yellow cocoon, white cocoon and Korea).

\begin{tabular}{|c|c|c|c|c|c|c|c|}
\hline No & Treatment & $\begin{array}{l}\text { Number of } \\
\text { double and } \\
\text { above/plot }\end{array}$ & $\begin{array}{c}\text { Average } \\
\text { weight of } 10 \\
\text { cocoons }\end{array}$ & $\begin{array}{c}\text { Defective } \\
\text { cocoon } \\
\text { percentage (\%) }\end{array}$ & $\begin{array}{c}\text { Reeling } \\
\text { quality (\%) }\end{array}$ & $\begin{array}{c}\text { Length of a } \\
\text { single } \\
\text { cocoon }\end{array}$ & $\begin{array}{c}\text { Silk } \\
\text { ratio (\%) }\end{array}$ \\
\hline 1 & $3 \mathrm{~cm} \times 3 \mathrm{~cm}$ & $1.65 \pm 0.33 d$ & $1.87 \pm 0.31 a$ & $0.00 \pm 0.00 \mathrm{~b}$ & $91.79 \pm 1.25 a$ & $674.33 \pm 10.17 c$ & $21.26 \pm 0.32 b c$ \\
\hline 2 & $3 \mathrm{~cm} \times 4 \mathrm{~cm}$ & $2.00 \pm 0.00 d$ & $2.67 \pm 0.17 a$ & $0.00 \pm 0.00 b$ & $94.50 \pm 1.21 a$ & $933.67 \pm 35.17 b$ & $23.59 \pm 0.83 a$ \\
\hline 3 & $3 \mathrm{~cm} \times 5 \mathrm{~cm}$ & $14.00 \pm 1.52 c$ & $2.97 \pm 0.05 a$ & $0.39 \pm 0.01 a$ & $67.57 \pm 1.21 b$ & $1002 \pm 17.03 a$ & $19.13+0.43 d$ \\
\hline 4 & $4 \mathrm{~cm} \times 4 \mathrm{~cm}$ & $2.00 \pm 0.57 d$ & $2.69 \pm 0.09 a$ & $0.00 \pm 0.00 b$ & $94.03 \pm 0.98 a$ & $987.67 \pm 11.69 b$ & $22.65 \pm 0.74 \mathrm{ba}$ \\
\hline 5 & $4 \mathrm{~cm} \times 5 \mathrm{~cm}$ & $23.54 \pm 2.60 b$ & $3.08 \pm 0.06 a$ & $0.40 \pm 0.03 a$ & $64.92 \pm 1.80 \mathrm{~b}$ & $979 \pm 25.69 a$ & $19.63 \pm 0.42 d c$ \\
\hline 6 & $5 \mathrm{~cm} \times 5 \mathrm{~cm}$ & $28.63 \pm 1.20 a$ & $2.90 \pm 0.02 a$ & $0.43 \pm 0.01 a$ & $66.11 \pm 3.28 b$ & $1023 \pm 5.89 a$ & $18.62 \pm 0.49 d$ \\
\hline & CV\% & 21.24 & 10.30 & 12.56 & 3.88 & 3.91 & 4.81 \\
\hline
\end{tabular}

Means followed by the same letter within a column are not significantly different from each other at $1 \%$ level of probability

(Student-Newman- Keul’s Range Test). 
Ahmed lbrahim et al.,

Mounting and mountages considerably influences the quality of cocoons. The farmers are said to be losing about $5-8 \%$ of yield due to improper mountages (Chandrakanth et al., 2004). It is evident from the mean data of the experiment that in general, ply wood, cartoon and banana leaf mountages showed a marginal tendency to improve many of the economic character as compared to the plastic and rolling paper montage. The results of the present study correlate with (Chandrakanth et al., 2004) where the author used seven types of mountages: Banana leaf type, mango twigs type, shoot rearing rack rotary type, plastic collapsible, fixed vertical type, bamboo mountages and rotary mountages considering cocooning $(\%)$, double cocoon(\%), floss $(\%)$, defective cocoon (\%), single cocoon weight $(\mathrm{gm})$, shell weight $(\mathrm{gm})$, shell ratio $(\%)$, and reel ability (\%) in which the bamboo mat base easily available, cheap and can last $4-5$ years but demerit using lot of space during mounting but both all of these mountages shows some merits as well as demerits during study time and also shows variations in economic parameters of cocoon production and quality in each type of mountages. Datta Biswas et al. (2008) also shows Plastic collapsible montage, plywood montage, mango and banana leaf mountage are an alternate to bamboo spiral and others mountage in Eastern India for better cocoon yield. Chikkanna et al. (2009) also study qualitative improvement in terms of economic gained by using more than two different types of mountages for silkworm cocoon. He also quote that, types of mountages, sizes and mounting environmental condition play a paramount role in determining the quality of cocoons of silkworm, Bombyx mori. Pandey et al. (2007) indicated that, plywood and cartoon made mountages with $4 \times 5 \mathrm{~cm}$ for Eri silkworm larvae and $4 \mathrm{~cm} \times 4 \mathrm{~cm}$ and $4 \mathrm{~cm} \times 3 \mathrm{~cm}$ for mulberry silkworm mounting space sizes in North-western India showed better result during study period. Datta Biswas et al. (2007) also showed comparative study of spinning of silkworm in more than three types of mountages (mango leaf, carton made, rolling paper, plastic mountage and banana leaf made montage). The Mango tree twigs and banana leaf mountages are playing important role in saving the cocoon crop and are easily available and easy to use for farmers but it has some major draw backs that during harvesting the dried leaves some times stickup to cocoons and the cost of twigs cannot be calculated. According to Mathur and Quadri, (2010) also estimated that farmers loss about $12-15 \%$ of crop due to defective cocooning which is attributed to inadequate Mountages, poor quality of Mountages, shortage of time, lack of proper mounting space, mounting care and management of environmental conditions. Our studies confirmed that, the percentage of urinated cocoon was more in plastic and rolling paper mountage compared to other types of mountages. Pandey et al. (2007) also used banana and mango leaf mountage in North-Western India which shows better results in improving cocoon quality during study period.

Thus from the present study, it can be concluded that the mountage made from plywood, Mango tree twigs, banana leaf and cartons are directly placed on rearing bed to spin cocoon helping the farmer to save labor and do not have any problems of identifying and picking ripe larvae at newly joined sericulturist. However, this method
Sci. Technol. Arts Res. J., April-June 2015, 4(2): 48-52

needs more space but during emergency time when there were no sufficient mountages or no any mountages with poor, newly joined farmers at that time Mango tree twigs and banana leaf mountages play vital role for farmers to save cocoon crop.

\section{CONCLUSIONS}

Therefore from the present study, it can be concluded that, plywood made, carton made and banana leaf made mountage followed by mango leaf mountage types should be recommended for eri and mulberry silk worms. Regarding the mountage sizes, $4 \mathrm{~cm} \times 4 \mathrm{~cm}$ and $4 \mathrm{~cm} \times$ $5 \mathrm{~cm}$ mountage size made from ply wood should be recommended for mulberry and Eri- silkworms, respectively, and used by the end users.

\section{Conflict of Interest}

Conflict of interest none declared.

\section{REFERENCES}

Chandrakanth, K.S., Srinivasa Babu, G.K., Dandin, S.B., Mathur, V.B., Mahdevmurthy, T.S. (2004). Development of improved Mountages. Indian Silk 43: 7-11.

Chikkanna, G.S., Singh, V.A and Qadri, S.M.H. (2009). Qualitative and improvement in terms of economic gained by using two different types of Mountages for Silkworm cocoon. Green Farming 2(14): 1014-1016.

Datta Biswas, T., Saha, A.K., Das, S.K. and Sarkar, S. (2007). A comparative study of Spinning of silkworm in two types of Mountages. Indian Sericulture 11(2): 39-43.

Ganga, G. and Sulochana Chetty, J. (1997). Hand book of Silkworm rearing Technology.

Hiware, C. J. (2001). Agro-Cottage Industry Sericulture. Daya Publishing House, Delhi, India. Pp. 57-93.

Inokuchi, T., Singh, G.B., Meenal, R., Rajan, R.K., Himantharaj, M.T. (1995). Evaluation of mountages for shoots rearing (JICA), project no. CSRM 1227.

Krishnaswami, S. (1978). New technology of silkworm rearing. Central Sericultural Research and Training Institute, Mysore, India. Pp:1-28.

Krishnaswami, S. (1978). New Technology of Silkworm rearing. Central Sericultural Research and Training Institute, Mysore, India. 57-93.

Mathur, V.B. and Qadri, S.M.H. (2010). Manual on Mountages, Mounting and Harvesting Technology for Quality Cocoon Production, C.S.R. and T.I., Central silk Board, Mysore.

Naphade, S.T., Hiware, C.J. and Avhad, S.B. (2010). Development of improved Mountage Using Mango plant twigs during lack of sufficient number or absence of Mountages on field for Silkworm cocoon. Recent Research in Science and Technology 2(7):05-08.

Pandey, R.K., Khan, M.A., Bindroo, B.B., Dhar, A. and Chauhan, S.S. (2007). Plant shoots Mountages of NorthWestern India. Indian Silk 46(8):4-5.

Vindhya, G.S., Bipin Kumar and Gangadhar, B. (1985). Mounting trials with different types of mountages project no. CSRM 1190. 